'Proyecto Kefuri, Web Intelligence Centre, Facultad de Ciencias Físicas y Matemáticas, Universidad de Chile. Santiago, Chile.

${ }^{2}$ Departamento de Medicina Oriente, Facultad de Medicina, Universidad de Chile; Unidad de Nefrología, Diálisis y Trasplante Renal, Hospital del Salvador. Santiago, Chile.

angeniero Civil Industrial.

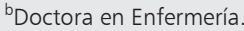

Trabajo no recibió financiamiento. Autores declaran no tener conflictos de intereses.

Recibido el 18 de noviembre de 2019, aceptado el 30 de enero de 2020.

Correspondencia a: Francisca González Cohens Domeyko 2367, Santiago, Chile. Francisca.gonzalez@ug.uchile.cl

\section{Análisis crítico de la baja tasa de donación de órganos en Chile}

\author{
FRANCISCA GONZÁLEZ COHENS ${ }^{1, \mathrm{a}}$, FELIPE VERA CID ${ }^{1, \mathrm{a}}$, \\ ROSA ALCAYAGA DROGUETT ${ }^{1, b}$, \\ FERNANDO GONZÁLEZ FUENZALIDA ${ }^{2}$
}

\section{Critical analysis of the low organ donation rates in Chile}

The highest organ donation rate in Chile was 10 donors per million population in 2017, which is low compared with other countries. Local experts attribute the low rate to population's lack of education and generosity, distrust in procurement/allocation system, inadequate legislation and insufficient encouraging campaigns, although without much empirical support: None of the countries from the OECD is also an organ donation leader. Latin Americans who migrate to Spain improve their donation rates, suggesting that the possible explanation is trust in the system where personalities do not have fast access to organs and common people do not have a sense of inaccessibility. Legislation changes in Chile did not have the expected results. Mega campaign did not reduce family refusal, but increased actual donors, probably due to health personnel sensitization. Real problems are inefficiencies in search and procurement processes, because procurement coordinators do not have enough time, dedication or priority to detect possible donors. Eighty seven percent of the latter are not notified to the procurement coordinator. Also, the services that care for possible donors are not adequately aligned. Procurement nurses do not have enough empathy or communication abilities and do not fulfil the professional profile required by the national coordination entity, which is unable to demand for results. The management of procurement coordinators should be improved, and their operational limitations should be visualized. Tools should be provided to the national agency in charge of organ procurement to have more political influence and credibility. Information technologies could ease warns, control and standardize, in real time, the procurement process.

(Rev Med Chile 2020; 148: 242-251)

Key words: Organ Transplantation; Tissue and Organ Procurements; Transplants.
L a tasa de donación de 6,6 donantes por millón de población (dpmp) de $2018^{1}$ fue atribuida a un impedimento regulatorio de preguntarle a familiares de fallecidos por su Testimonio de Última Voluntad (TUV) si se encontraba en el Registro Nacional de No-Donantes. Esa cifra fue muy distante a la "histórica" de 2017 (10 dpmp) que alentó a muchos a pensar de que finalmente Chile iba bien encaminado ${ }^{1}$, incluso autoridades gubernamentales arguyeron que el éxito obedecía a mejoras en procesos y maduración del sistema de donación y trasplantes ${ }^{2}$.

Lo más mediático de la época fue la campaña del canal Mega enfocada en sensibilizar la población $^{3}$ y que, a decir de sus encargados, lograría reducir la negativa familiar (NF) a donar (Infor- 
mación personal director campaña). No obstante, 10 dpmp sigue siendo bajo, más aun considerando que se encontraba dentro de lo proyectable desde años previos sin mediar campañas en pro o contra a la donación ${ }^{4}$.

\section{Objetivo}

Mejorar la donación depende de conocer a fondo las causas subyacentes a las bajas tasas. Pretendemos compartir y explicar algunas de ellas y proponer alternativas de mitigación o solución.

\section{¿Por qué Chile tiene tasas tan bajas de donación?}

Expertos locales, sin necesariamente sustento empírico, postulan: Falta de educación o generosidad poblacional, desconfianza con el sistema de procuramiento/asignación de órganos, legislación inadecuada y falta de campañas comunicacionales que incentiven la donación ${ }^{5-7}$. Casi nadie sostiene que el problema real es que los procesos de pesquisa y procuramiento son ineficientes.

Veamos datos para responder cada una de esas hipótesis:

\section{a) Falta de educación en la población}

Las sociedades más educadas entenderían mejor el significado de muerte, enfermedad y tratamientos médicos y, por tanto, son o debieren ser más proclives a donar órganos. No obstante, ninguno de los países con mejores índices de educación es líder en donación (Figura 1$)^{8,9}$ y distan del líder, España.

Aunque no muy diferente de Chile en el aspecto educacional, España ha logrado alzas en sus tasas de donantes a velocidades superiores a las requeridas para conseguir cambios culturales o educacionales poblacionales: 14 dpmp 1989, 28 dpmp 1997 y 42 dpmp 2016 (Figura 2) ${ }^{10}$.

\section{b) Falta de generosidad en la población}

La donación en Latinoamérica es muy inferior a la española, aunque, si se compara las tasas de emigrantes latinoamericanos a España con las tasas en sus países de origen, se ve crecen aparentemente "sin explicación"; ejemplos, Colombia tiene localmente una tasa de donación de 12,3 y sube a $44,5 \mathrm{pmp}$ en los colombianos emigrados a España, Ecuador sube de 2,2 a 38,8 y Chile de 5,4 a 33, $9^{11}$.

Por tanto, la generosidad no parece ser el pro-

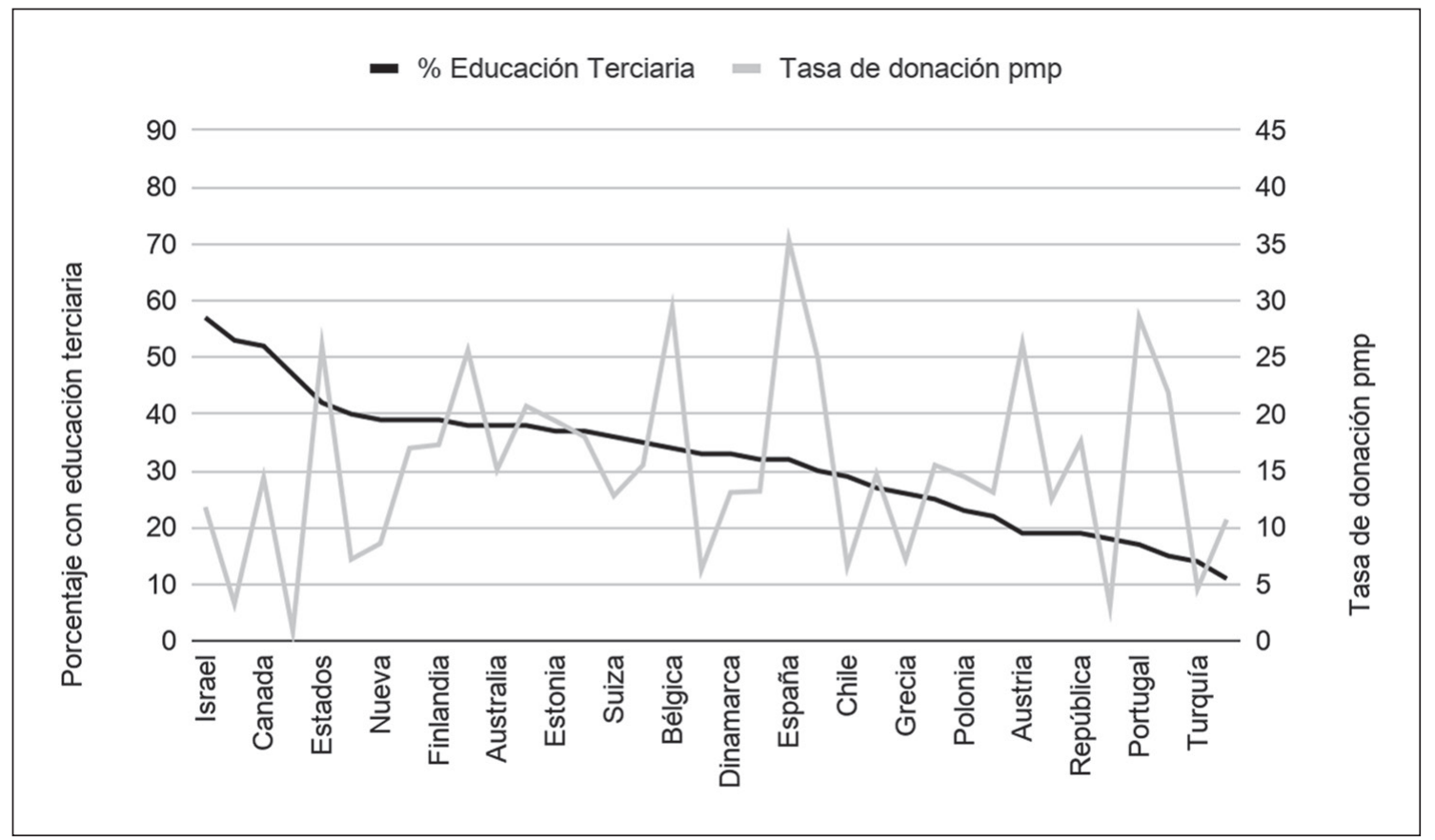

Figura 1. Proporción de la Población que ha alcanzado Educación Terciaria comparado con la tasa de donantes efectivos pmp por países OCDE (2011) (Adaptado de ${ }^{8,9}$ ). 


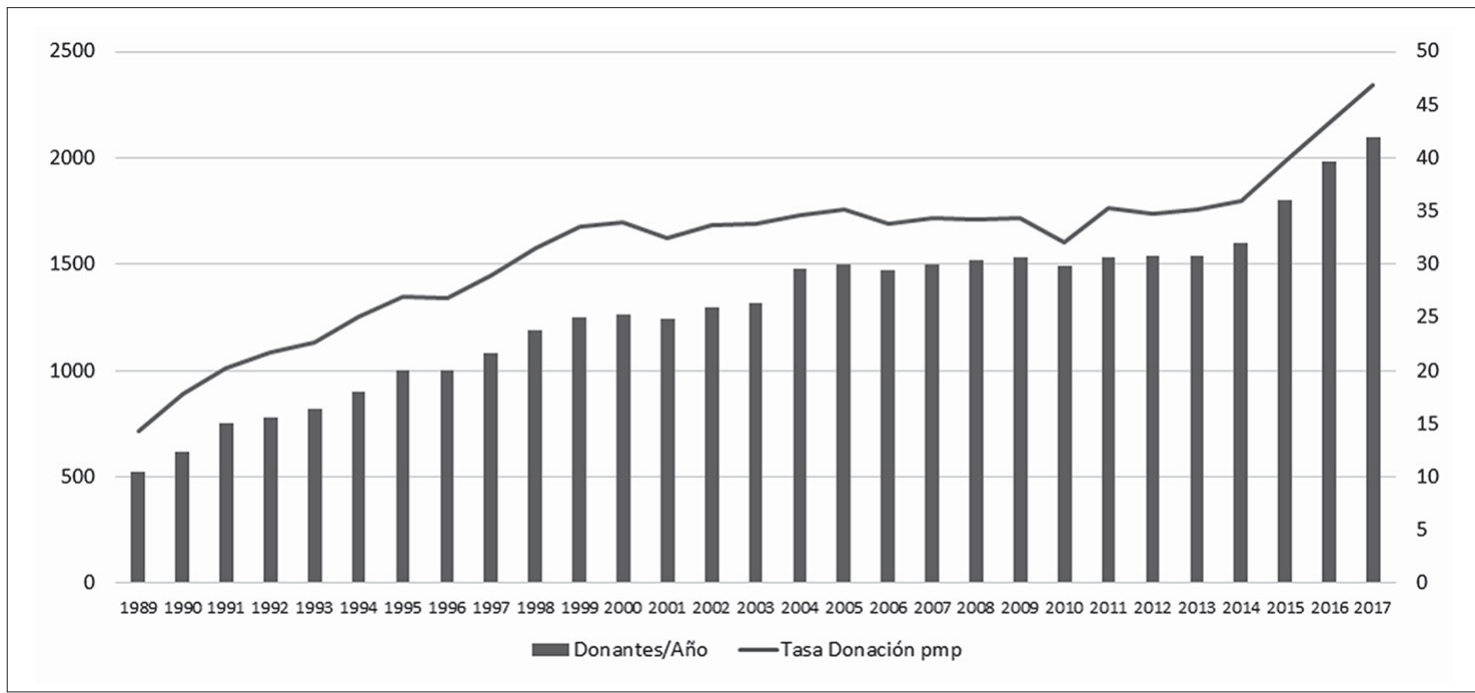

Figura 2. Evolución donantes (eje vertical izquierdo) y tasa donación pmp (eje vertical derecho) en España (1989-2017) (Adaptado de ${ }^{10}$ ).

blema, sino que de alguna forma, la confianza en el sistema de salud español es inmensamente superior a lo percibido en Latinoamérica ${ }^{12}$.

\section{c) Desconfianza con el sistema de procuramien- to/asignación de los órganos}

Aunque no medido formalmente, los casos mediáticos de personas trasplantadas o que no pudieron serlo impactan la opinión pública:

i. Los trasplantes renales de ministros (Agricultura 2007 e Interior 2009) y cónyuge del de Salud (2011) pusieron en entredicho la equidad del trasplante. Ninguno de los dos primeros se dializaba al momento de ser intervenidos y la última recibió un trasplante pulmonar, tampoco accesible para la mayoría poblacional ${ }^{4}$.

ii. La inaccesibilidad a la lista de espera de corazón de una niñita del Servicio Nacional de Menores (2017) perturbó a la población chilena ${ }^{5}$, así como el que se destaque pacientes económicamente aventajados o alcohólicos recibiendo trasplantes ${ }^{4}$.

iii. Algo similar ocurre cuando se pone en duda el financiamiento de los trasplantes o que no exista tráfico de órganos ${ }^{5,15}$.

iv. La sensación general sería que se privilegia a personas con poder y dinero y no a quienes más lo necesitan. Efectivamente, aunque el sistema público genera más donantes que el privado, no trasplanta más. Peor aún si se considera los órganos no-riñón, se trasplantan muchísimo más en el sistema privado debido a la incapacidad del sistema público de brindar atención a ciertas enfermedades terminales provocando migración de un sistema al otro ${ }^{15}$.

\section{d) Marco legal inadecuado}

La Ley de Trasplante en Chile ha evolucionado, favoreciendo dicha actividad ${ }^{16}$.

i. Ley 19.451 (1996): Donación gratuita, los órganos pueden provenir de donantes vivos relacionados o de no-vivos (fallecidos) que hayan manifestado su voluntad o que sus familiares consientan en ello.

En 1996 el elemento “complejo" era el concepto de muerte encefálica, su validez científica y ética, su diagnóstico y las posturas religiosas, éticas, jurídicas y sociales que la sustentaban. El énfasis era explicar y convencer a los familiares de alguien fallecido encefálicamente a donar los órganos del ser querido.

ii. Ley 20.413 (2010): Definición de Donante no-vivo presunto, es decir, todos somos donantes a menos que en vida hayamos manifestado lo contrario. Crea la Coordinadora Nacional de Procuramiento y Trasplante (CNPT), encargada de elaborar la política y normas respectivas.

Se generó un "Registro de No-Donantes" de 3,9 millones de personas registradas renovando 
cédula de identidad o licencia de conducir. Paralelamente, se continuó preguntando a familiares por la voluntad del fallecido, estuviera o no en el Registro.

iii. Ley 20.673 (2013): Confirma el "Donante presunto" y establece que el documento de registro como no-donante será notarial y elaborado con anterioridad al fallecimiento de la persona. Su Reglamento (Decreto 35) impone la obligatoriedad del médico tratante de notificar la potencial muerte encefálica a la Coordinación Local de Procuramiento (CLP). Por primera vez, un Reglamento releva el proceso de detección y manejo del potencial donante, originando el proceso de procuramiento y existencia de unidades de coordinación de procuramiento a través de la creación de la CNPT.

iv. Ley 21.145 (2019): Permite "limpiar" el registro de no-donantes y especifica que no se preguntará a la familia la voluntad de donar del fallecido, a menos que se encuentre en el registro nacional de no-donantes o exista duda fundada.
El Consentimiento Presunto supone que la tasa de donación aumenta respecto de cuando es autorizada por familiares, suposición no necesariamente cierta (Figura 3) ${ }^{17}$, de hecho, se observó un fuerte ascenso en la NF $(36 \% \text { a } 50 \%)^{18}$. A pesar de que el procuramiento fue institucionalizado, no aparecieron diferencias significativas ni en cantidad de donantes ni de pesquisas, sugiriendo que no hubo cambios sustanciales (Figura 4).

Es importante mencionar también que el Dictamen de Contraloría, posible responsable de la caída de donación de 2018, tampoco representa un punto de inflexión destacable, ya que, a pesar de haber diferencias significativas en pesquisas y donantes durante el período 2013-2018, el año que realmente contribuye a esa diferencia es 2017 y no $2018^{19}$.

\section{e) Falta de campañas comunicacionales que incentiven la donación}

Siempre aparece como relevante en las discusiones del tema. Aunque el Ministerio de Salud ha implementado campañas acotadas no se conoce evaluaciones objetivas de su impacto real en las tasas de donación.

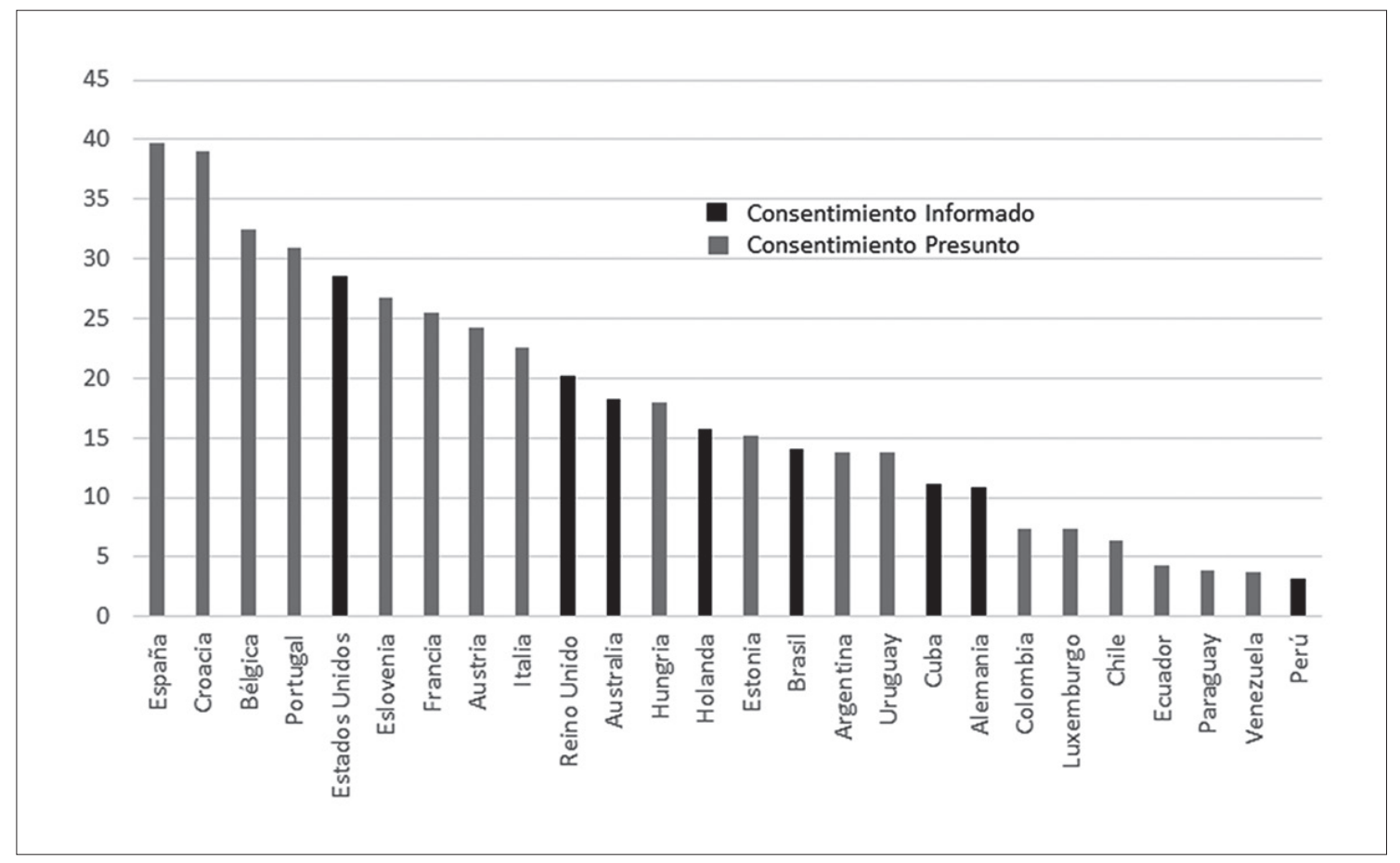

Figura 3. Tasa de donación en donantes por millón de población (eje vertical) en países con y sin consentimiento presunto a la donación de órganos (Adaptado de ${ }^{17}$ ). 


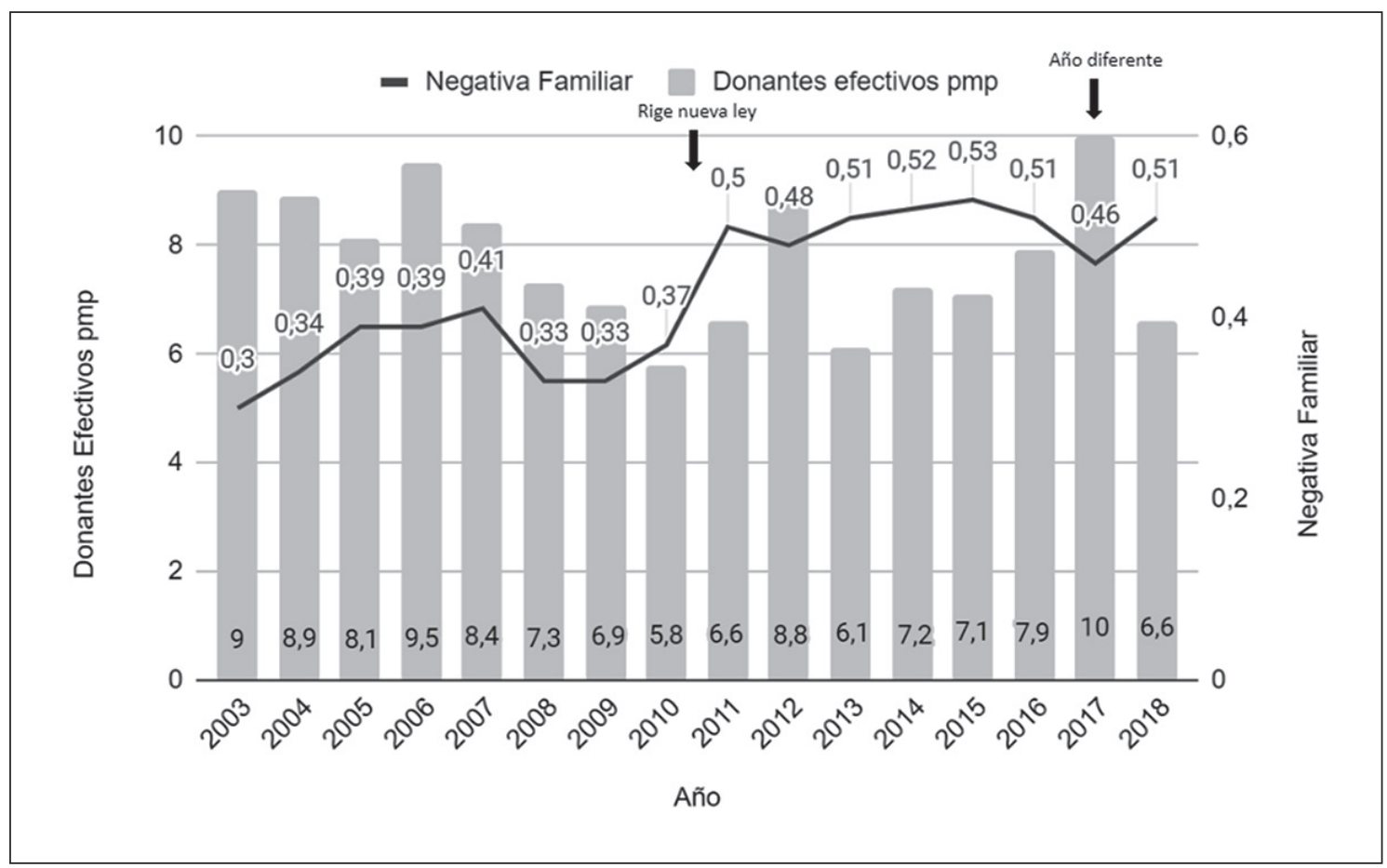

Figura 4. Efecto sobre la tasa de donación en Donantes por millón de población (pmp) y Negativa Familiar en Chile como consecuencia de la ley de consentimiento presunto del año 2010 (Adaptado de ${ }^{1,18}$ ).

Es sabido que las campañas sensibilizan a su población objetivo ${ }^{4,20,21}$, pero no es claro que efectivamente cambien las cifras de donación ${ }^{22}$.

La campaña Mi Causa Mi Mega (2016-17) buscó sensibilizar a la población y, por ende, reducir la $\mathrm{NF}^{3}$. Su resultado tras 12 meses de intensa exposición mediática fue decepcionante; la NF no varió significativamente ${ }^{23}$ aunque sí consiguió más donantes, tanto, que el año 2017 es considerado como el mejor año de donación de órganos ${ }^{4}$ (Figura 5).

Esta discordancia entre no reducción de la NFe incremento en la tasa de donación efectiva probablemente resultó de sensibilizar no a la población objetivo, sino que, realmente, a quienes trabajan en los hospitales donde se detectan y consiguen los donantes.

\section{¿Dónde está el verdadero problema entonces?}

Las causas subyacentes a la baja tasa de donación no parecen estar en la sociedad o, dicho de otro modo, en los potenciales donantes o sus familias. ¿Será que el proceso de procuramiento tiene fallas susceptibles de ser mitigadas o corregidas?

El proceso de donación puede subdividirse operacionalmente en varios subprocesos: Detección del posible donante $(\mathrm{PD})$ en servicios de urgencia (SU) o de paciente crítico (UPC), aviso de su existencia a la CLP, traslado del PD a una UPC para su estabilización hemodinámica y metabólica, diagnóstico de muerte encefálica, solicitud del TUV y, en caso de haber consentimiento de la donación, coordinación y logística de la cirugía de extracción y traslado de órganos y tejidos a instituciones implantadoras. Todos bajo el control y alero organizacional de la CNPT.

\section{a) Estructura del sistema}

El modelo Español (líder mundial con 46 dpmp en 2017) ${ }^{9}$ y referente e inspirador de muchos otros países se basa y sustenta en una red de coordinadores en 3 niveles: Nacional, Autonómico y Hospitalario. Los primeros constituyen una interfaz entre los niveles políticos/adminis- 


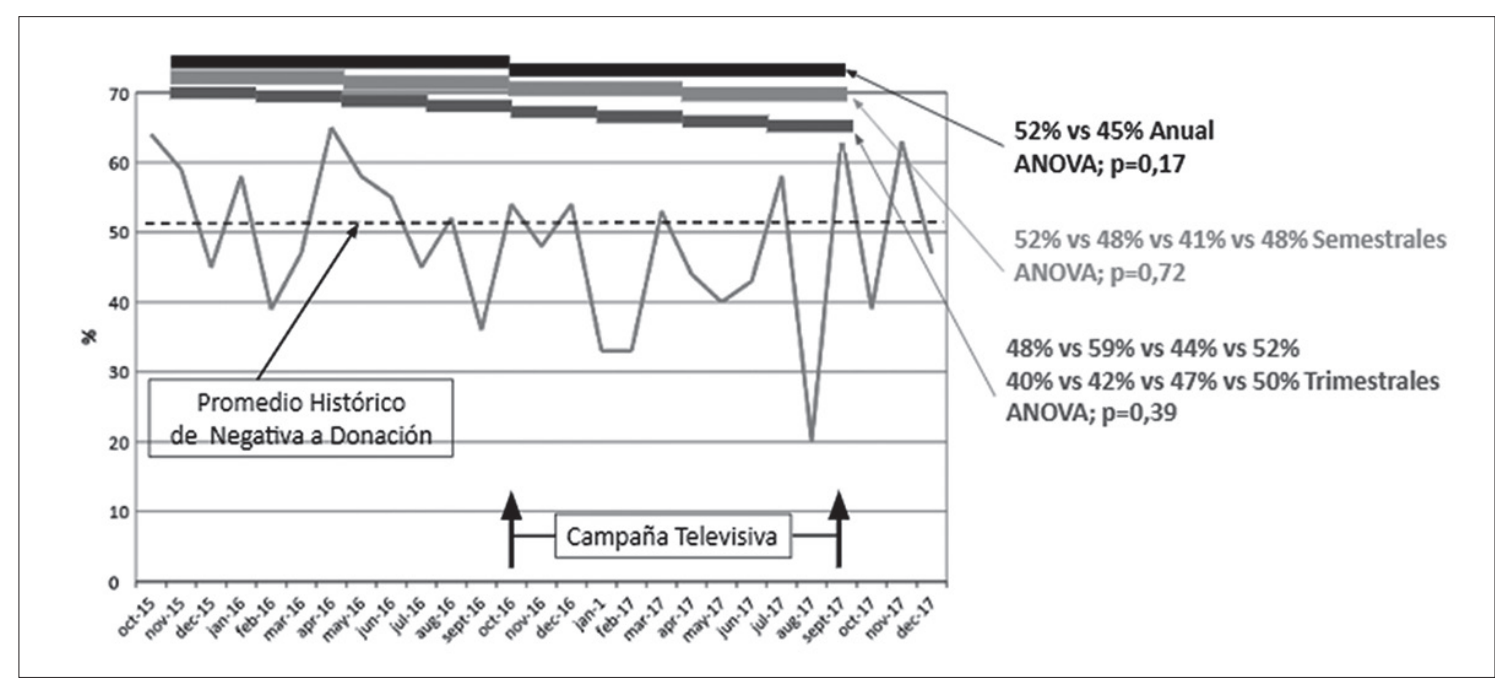

Figura 5. Negativa familiar a la Donación de Órganos en Chile. Período octubre de 2015 a diciembre de 2017 (Adaptado de ${ }^{23}$ ).

trativos y profesionales y el tercero, en coordinadores hospitalarios médicos, generalmente intensivistas, que trabajan tiempo parcial en la coordinación con colaboración de enfermeras a tiempo completo ${ }^{24}$.

Chile adaptó esa estructura general instalando coordinaciones en los mismos 3 niveles, pero las CLP, presentes en 27 de los 29 Servicios de Salud del país, quedaron dirigidas por enfermeras no siempre con dedicación laboral exclusiva ni, tampoco, a tiempo completo ${ }^{25}$.

\section{b) Detección del PD}

Lo complicado de la pesquisa es que depende de la cooperación y aviso de quienes trabajan en SUs y que, además, desconocen que incluidas en sus tareas está el avisar de la presencia de un PD (Dto. 35, Ley 19.451) ${ }^{16}$. La relevancia de esta etapa se desprende de la constatación (2016), en 2 hospitales, que $60 \%$ de los PD se habían perdido en ella (Información personal José Luis Rojas, Coord. Nac.), cifra que puede ascender incluso al $87 \%{ }^{26}$. En otras palabras, sólo $13 \%$ de los PD son pesquisados (inician seguimiento por parte de las CLP). De éstos, el 10\% se pierde por contraindicación médica, dejando que $11,6 \%$ de los PD llegan a la STUV, donde, debido a la NF de $\sim 50 \%{ }^{27}$, únicamente el $5,3 \%$ de los PD iniciales se convierten en donantes efectivos.

Revertir las malas cifras no depende de la voluntad de la gente a donar sino principalmente de mejorar la efectividad de los procesos de detección, procuramiento y mantención del $\mathrm{PD}^{26}$.

\section{c) Aviso del PD a quienes coordinan localmente la procura}

El modelo español incluye un programa de calidad consistente en auditorías continuas de las muertes encefálicas en las UPCs, permitiendo, mediante indicadores, analizar fugas y definir mejoras, siendo primordial la capacidad hospitalaria de detección sistemática de todo paciente que fallezca encefálicamente ${ }^{24}$.

Gran parte de su éxito se basa en que sus CLP se enteran rápidamente de la existencia de PDs, convirtiendo la comunicación eficaz en estratégica. En Chile, encuestas al personal clínico de SU, ilustran que aunque todos conocen de la actividad de procura y donación, pocos afirman conocer cómo detectar o avisar de la presencia de PDs. Desconocen la existencia y labor de las CLP, sus roles de turno, disposición en horas no diurnas o cómo contactarlas ni quién es el responsable de hacerlo. Pocos declaran conocer la obligación legal de avisar de la presencia de un PD. Sí concuerdan la mayoría, en la dificultad de trasladar al PD a UPCs ${ }^{28}$.

\section{d) Traslado del PD a UPC para estabilización y mantención hemodinámica y metabólica}

Aunque los $\mathrm{PD}$ son pacientes graves ( $<7$ puntos en la escala de Coma de Glasgow y diagnóstico 
objetivo de una catástrofe intracraneal, como traumatismo o accidente vascular encefálico) no todos acceden a UPC, dificultad que es destacada por el personal de $\mathrm{SU}$, según encuestas realizadas en SU y UPC, donde estos últimos afirman que, de ser posible, ingresan prioritariamente a PDs para brindarles el tratamiento necesario ${ }^{28}$.

No excepcionalmente, quienes laboran en estas unidades desconocen si un PD necesita de cuidados especiales para mejorar el estado funcional de los órganos que se espera done; en ese aspecto, las CLP pueden colaborar coordinando la comunicación entre especialistas ${ }^{29}$.

\section{e) Diagnóstico de muerte encefálica}

Este punto parece el menos crítico desde que las garantías explícitas en salud del accidente vascular encefálico, obliga a contar con tomografía axial computarizada de cerebro y un neurólogo evaluador $^{30}$.

La Ley establece que la forma de certificar la muerte encefálica es vía un Test de Apnea realizado por especialistas no relacionados al equipo de trasplante sin necesidad, salvo excepciones, de realizar exámenes diagnósticos complementarios ${ }^{16}$.

\section{f) Solicitud de testimonio de última voluntad (STUV)}

Es la etapa más sensible del proceso de procuramiento. El que sea exitosa no depende únicamente de la voluntad del PD o familiares, sino, también, de un proceso claro, correcto y transparente que informe periódicamente de la gravedad, mala evolución y seguro desenlace del paciente a la familia. No debe hablarse de donación antes de existir clara comprensión del concepto de muerte encefálica como fin de la vida o antes que CLP lo aclaren por completo.

Dado que el objetivo de una STUV es obtener una venia a la extracción de órganos, la CLP facilita la ayuda emocional para iniciar su duelo enmarcándolo en una perspectiva de generosidad y solidaridad. Resulta obvio que las "habilidades blandas" y preparación técnica y sicológica de las CLP sean cruciales, características que no todas ellas poseen ${ }^{25}$.

\section{g) Coordinaciones Locales de Procuramiento}

El procuramiento es complejo e involucra a diferentes actores, personal de SU (pesquisa), UPC (mantención) y de equipos quirúrgicos, coordinados todos por la CLP, sea médico ${ }^{31}$ o enfermera ${ }^{32}$.
Los desempeños de las CLP son estadísticamente distintos, tanto en la cantidad de PD pesquisados como en sus donantes efectivos y sus $\mathrm{NF}^{33}$ : Cada CLP rinde de forma diferente con respecto a sus pares. Algunas son relativamente buenas en pesquisa ("alta" cantidad de PD) y otras en la STUV (baja NF). No hay claridad de la razón que algunas sean buenas en lo que son y tampoco el por qué son deficientes aquéllas que tienen peores resultados. Favorecerían mejores resultados el contar con varias profesionales y que compartan labores, de alguna forma, con unidades de trasplante, ya sea en coordinación o en controles pre o postrasplante ${ }^{34}$.

No todas las CLP poseen perfil profesional idóneo: Experiencia en Urgencia (18\%) o UPC $(41 \%)$, capacitación adecuada $(80 \%<7$ días $)$ y formación en donación $(34 \%)^{25}$. Pocas fidelizan al personal de Urgencias (indispensable para detectar donantes), desconocen cómo mantener un PD en UPC, no se dan a conocer como referentes en el tema dentro del ámbito laboral y tampoco cuentan con un proceso de procura técnico-estructurado-normalizado, ni mecanismos de evaluación de procesos $^{25}$.

Las características de las CLP pretenden explicar, parcialmente, su dispar desempeño. Dicha heterogeneidad es la constante en el tiempo para las unidades, es decir, casi todas muestran desempeños anuales irregulares. Da la impresión que las cifras anuales de pesquisa, NF y donación efectiva son gobernadas principalmente por el azar y no por trabajo que muestre mejora continua ${ }^{35}$.

El problema subyacente a esto último es que la CNPT no tiene herramientas para controlar la gestión de las CLP, y, por ende, le impide identificar los determinantes de éxito y si ello puede replicarse a lo largo del país ${ }^{35}$.

\section{h) Coordinadora Nacional de Procuramiento y Trasplante del Minsal}

La actividad de procura es aleatoria y, consecuentemente, no refleja mejora continua como propendería el trabajo de la CNPT (Figura 6) ${ }^{4}$.

Chile no posee un programa de calidad, sólo se sugiere etapas del proceso y no es normativo, por tanto, renuncia a su supervisión y seguimiento ${ }^{25}$. Incluso, como las CLP son contratadas por el hospital mismo, deben cumplir las funciones que el director local les asigna y que no necesariamente están alineadas con los objetivos de la CNPT. 


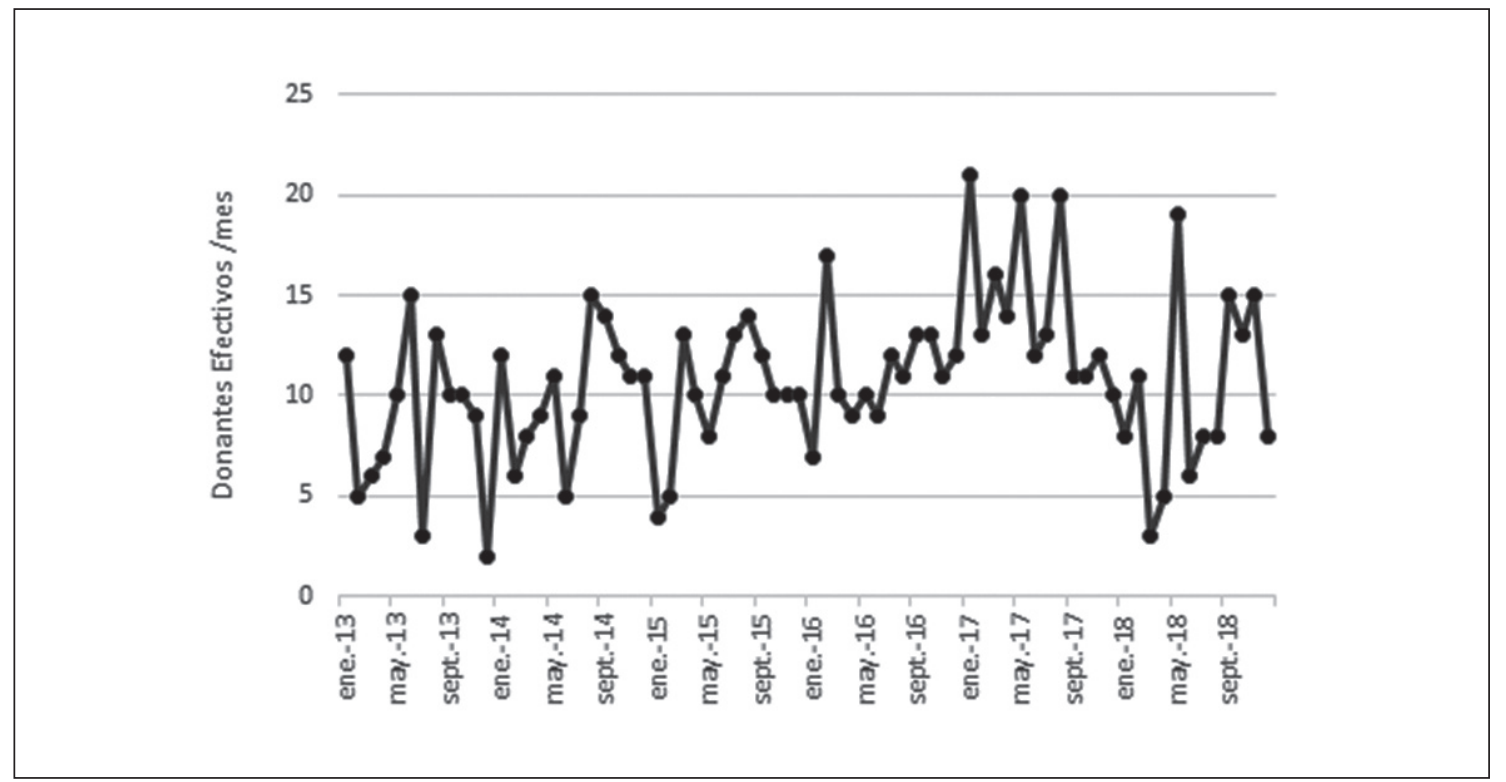

Figura 6. Donantes efectivos período enero de 2013 a diciembre de 2018 (Adaptado de Información SIDOT 2013-2018 proporcionada por Dr. José Luis Rojas, Coord. Nac.).

El impedimento real para mejorar la donación obedece a:

- La CNPT carece de suficiente poder político, económico y administrativo para llevar a cabo su única misión: "Implementar una política nacional de donación y trasplante en el marco de las normas, objetivos y principios establecidos en la normativa vigente"16.

- Control insuficiente de procesos hospitalarios.

La escasa fiscalización impide a autoridades el visualizar y solucionar los problemas ${ }^{36}$. No se destina recursos ni voluntades para hacerlo, lo cual emana de observar el escaso presupuesto y poder político y administrativo que tiene la CNPT dentro el aparato sanitario ${ }^{37}$ y hace imperativo el buscar soluciones como serían la automatización y control de procesos y desempeños.

Controlar los procesos y visibilizar sus "cuellos de botella", en cada centro, permitiría entregar herramientas fidedignas a la CNPT para mejorar su credibilidad e influencia ${ }^{38}$. Tecnologías al servicio de tales procesos, como son las derivadas de las tecnologías de la información (TI) permitirían aviso expedito de $\mathrm{PDs}^{39}$, estandarización de proceso de procura ${ }^{40} \mathrm{y}$ registro en tiempo real del desempeño de las CLP.

\section{¿Cómo cambiar la historia y mejorar las magras tasas de donación?}

Nuestra tasa de donación es baja y continuará siéndolo de no mediar cambios sustanciales:

1. Abandonar la creencia que es la Sociedad que no desea donar y dejar de insistir en iniciativas educacionales, particularmente si ellas serán insostenibles en el tiempo.

2. Convencernos que las campañas comunicacionales eficaces son extraordinariamente onerosas $\mathrm{y}$, a menos que se prolonguen en el tiempo, de muy corta duración en la memoria colectiva.

3. Lo que sí impacta en la población son las historias humanas, tanto de éxito como de fracaso. Debe buscarse el cómo destacar las primeras por sobre las otras.

4. Al ser una obligación del médico tratante avisar de la presencia de un PD, debería fiscalizárselos y sancionarse el no cumplimiento.

5. Facilitar comunicación entre los actores de la procura. Las TI, ciertamente, ayudarían al personal a dar aviso y cumplir con su obligación legal y a los fiscalizadores realizar su labor.

6. Reclutar, capacitar, estandarizar, controlar y auditar, en tiempo real, el trabajo de las CLP mediante las mismas TI. 
7. Desarrollar e implementar estrategias que faciliten la resolución de "cuellos de botella" operacionales que dificultan los procesos que concluyen en donaciones efectivas.

En síntesis, el futuro de la procura y, en último término, la actividad de trasplante de órganos donados generosamente por quienes fallecen encefálicamente de forma prematura (y tal vez, más adelante también de muerte cardíaca) pasa por hacer eficientes los procesos e incorporar herramientas de la ingeniería a las actividades clínicas y por capacitarnos mutuamente quienes laboramos en los campos de la medicina y la ingeniería.

\section{Referencias}

1. Ministerio de Salud. Yo Dono Vida - Estadísticas [Internet]. Ministerio de Salud - Estadísticas. [citado 26 de marzo de 2019]. Disponible en: https://yodonovida. minsal.cl/estadisticas/estadisticas.html

2. Maldonado RP. Salud destaca cifra récord de trasplantes de órganos en 2017: Se realizaron 457 [Internet]. La Nación. [citado 2 de abril de 2018]. Disponible en: http://lanacion.cl/2018/01/20/salud-destaca-cifra-record-de-trasplantes-de-organos-en-2017-se-realizaron-457/

3. Mega.cl. ¡Súmate a una causa comprometida con la donación de órganos! [Internet]. www.mega.cl. [citado 2 de abril de 2018]. Disponible en: http://www.mega.cl/ causas/informate/33655-donacion-de-organos.html

4. González Fuenzalida F, González Cohens F. El sinsabor que deja el mejor año en la donación de órganos para trasplante en Chile. Rev Med Chile 2018; 146 (5): 547-54.

5. Revista Asodi - Asodi [Internet]. [citado 21 de enero de 2020]. Disponible en: https://asodi.cl/revista/

6. Erwin Buckel: «La negativa familiar sigue siendo la causa número uno de no donantes» - Duna 89.7 | Duna 89.7 [Internet]. [citado 21 de enero de 2020]. Disponible en: https://www.duna.cl/programa/aire-fresco/2018/06/05/ erwin-buckel-la-negativa-familiar-sigue-siendo-la-causa-numero-uno-de-no-donantes/

7. Vera M, Alegría L, Domínguez J, Rebolledo R, Dib M. Donación y trasplante de órganos: análisis y propuestas para avanzar hacia una cultura de donación. 2019; 22.

8. Organisation for Economic Co-operation and Development (OECD). Education at a glance 2013: OECD indicators. [Internet]. 2013. Disponible en: http://www. oecd.org/education/eag2013\%20(eng)- FINAL\%20 20\%20June $\% 202013$.pdf
9. IRODaT - International Registry on Organ Donation and Transplantation [Internet]. [citado 1 de abril de 2018]. Disponible en: http://irodat.org/

10. Newsletter 2017 de la Organización Nacional de Trasplantes de España [Internet]. [citado 17 de julio de 2019]. Disponible en: http://www.ont.es/Documents/ Datos20172018ENE11.pdf

11. Ríos A, López-Navas AI, Navalón JC, Martínez-Alarcón L, Ayala-García MA, Sebastián-Ruiz MJ, et al. The Latin American population in Spain and organ donation. Attitude toward deceased organ donation and organ donation rates. Transpl Int. 2015; 28(4): 437-47.

12. Lancet T. Organ donation depends on trust. The Lancet. 25 de junio de 2016; 387(10038): 2575.

13. Mostrador E. Padres de León Smith tienen deuda de $\$ 554$ millones con Hospital Clínico de la UC [Internet]. El Mostrador. 2016 [citado el 17 de julio de 2019]. Disponible en: https://www.elmostrador.cl/noticias/ pais/2016/05/24/padres-de-leon-smith-tienen-deudade-554-millones-con-hospital-clinico-de-la-uc/

14. Chile B del CN de. Tráfico de órganos en Chile: ¿Mito o realidad? - Observatorio Parlamentario [Internet]. Observatorio Bioética. 2016 [citado 17 de julio de 2019]. Disponible en: http://observatorio.bcn.cl/bioetica/noticias/trafico-de-organos-en-chile-mito-o-realidad

15. González Cohens F, Vera Cid F, Alcayaga Droguett R, González Fuenzalida F. La inequidad del sistema de procuramiento y trasplante puede explicar, al menos en parte, las bajas tasas de donación en Chile. En La Serena, Chile; 2019. p. 138. Disponible en: http://www.nefro.cl/ v2/docs/libro_congreso_nefrologia_2019.pdf

16. SALUD MD. LEY-19451 10-ABR-1996 MINISTERIO DE SALUD [Internet]. Ley Chile - Biblioteca del Congreso Nacional. 1996 [citado el 26 de marzo de 2019]. Disponible en: https://www.leychile.cl/Navegar?idNor$\mathrm{ma}=30818$

17. Chaparro GR. La presunción de la donación de órganos en Colombia: reflexiones para el debate. Rev Latinoam Bioét 2017; 17 (2): 92-106.

18. Domínguez J, Rojas JL. Presumed consent legislation failed to improve organ donation in Chile. Transplant Proc 2013; 45 (4): 1316-7.

19. González Cohens F, Vera Cid F, Alcayaga Droguett R, González Fuenzalida F. Ni las campañas a favor ni en contra de la donación de órganos afectan las tasas de donación. En La Serena, Chile; 2019. p. 13. Disponible en: http://www.nefro.cl/v2/docs/libro_congreso_nefrologia_2019.pdfla inequi

20. Alvaro EM, Siegel JT, Crano WD, Dominick A. A mass mediated intervention on Hispanic live kidney donation. J Health Commun 2010; 15 (4): 374-87. 
21. Salim A, Berry C, Ley EJ, Schulman D, Navarro S, Chan LS. Utilizing the media to help increase organ donation in the Hispanic American population. Clin Transplant. 2011; 25 (6): E622-8.

22. Salim A, Malinoski D, Schulman D, Desai C, Navarro S, Ley EJ. The Combination of an Online Organ and Tissue Registry With a Public Education Campaign Can Increase the Number of Organs Available for Transplantation. J Trauma 2010; 69 (2): 451-4.

23. González Cohens F, Vera Cid F, Alcayaga Droguett R, González Fuenzalida F. El ineficiente uso de recursos en campañas de donación. En México; 2019. Disponible en: https://www.stalyc2019.com/trabajos-cientificos

24. Matesanz R. El modelo español de coordinación y trasplantes. Madrid: Aula Médica; 2008.

25. Alcayaga R. Procuramiento de órganos en Chile, un análisis crítico desde un modelo de gestión de calidad [Tesis de Doctorado]. [Concepción, Chile]: Universidad de Concepción; 2019.

26. González Cohens F, Vera Cid F, Rojas Barraza JL, González Fuenzalida F. La negativa familiar tiene un rol marginal en la baja tasa de donación de órganos en Chile. En Chile, México; 2019. p. 77. Disponible en: http://congreso2019.sociedaddetrasplante.cl/assets/ libro-resumen-congreso-trasplante-2019.pdf, https:// www.stalyc2019.com/trabajos-cientificos

27. Ministerio de Salud. Datos Donantes de Órganos y Receptores Año 2016 [Internet]. 2017 ene 13; Chile. Disponible en: https://www.minsal.cl/wp-content/ uploads/2015/09/consolidado_2016_final.pdf

28. González F, Adaros N, Canales M, Stekel C, Vera F, Camino S, et al. Estudio del proceso de pesquisa de posibles donantes de órganos en centros trasplantadores de la Región Metropolitana. Resultados preliminares. En 2017.

29. Schein AE, Carvalho PRA, Rocha TS da, Guedes RR, Moschetti L, La Salvia JC, et al. Avaliação do conhecimento de intensivistas sobre morte encefálica. Rev Bras Ter Intensiva 2008; 20 (2): 144-8.

30. Subsecretaría de Salud Pública D de P y C de E técnica A. Accidente cerebrovascular isquémico en personas de 15 años y más [Internet]. 2013 [citado el 17 de septiembre de 2019]. Disponible en: https://www.minsal.cl/portal/ url/item/7222754637e58646e04001011f014e64.pdf

31. Elizalde J, Lorente M. Coordinación y donación. An Sist Sanit Navar 2006; 29: 35-43.

32. Palacios J, Jiménez O, Turu I, Belmar P, Rocha S, López M. El coordinador de trasplante: su importancia en el procuramiento de órganos en Chile. Rev Chil Cir 2001; 53: 257-61.

33. González Cohens F, Vera Cid F, Rojas Barraza JL,
González Fuenzalida F. Las unidades de procura de los hospitales públicos requieren de urgente reingeniería: El modelo actual definitivamente no sirve. En Pucón, Chile: Sociedad Chilena de Nefrología; 2018 [citado el 28 de marzo de 2019]. p. 92-3. Disponible en: https:// www.nefro.cl/v2/biblio/congresos/240.pdf

34. González Cohens F, Vera Cid F, Alcayaga Droguett R, González Fuenzalida F. Coordinadoras part-time compartidas con trasplante parecen ser quienes más procuran. En Chile, México: VII Congreso Chileno de Trasplante y XXV Congreso Latinoamericano y del Caribe de Trasplante; 2019. p. 73. Disponible en: http:// congreso2019.sociedaddetrasplante.cl/assets/libro-resumen-congreso-trasplante-2019.pdf; https://www. stalyc2019.com/trabajos-cientificos

35. González Cohens F, Vera Cid F, Alcayaga Droguett R, González Fuenzalida F. El sorprendente desempeño de las CLP explican los resultados de donación en Chile. Análisis del período 2013-2018. En Chile, México: VII Congreso Chileno de Trasplante y XXV Congreso Latinoamericano y del Caribe de Trasplante; 2019. p. 74. Disponible en: http://congreso2019.sociedaddetrasplante.cl/assets/libro-resumen-congreso-trasplante-2019.pdf

36. Adaros N. Propuesta de rediseño organizacional para las unidades encargadas de la detección de posibles donantes en hospitales [Internet] [Memoria de Título]. 2017. Disponible en: http://repositorio.uchile.cl/handle/2250/148506

37. Canales Mena ME. Capacidades de la Coordinadora Nacional de Trasplantes para garantizar el acceso oportuno a un órgano a quien lo necesite [Internet] [Tesis de Magíster]. [Chile]: Universidad de Chile; Disponible en: http://repositorio.uchile.cl/handle/2250/152129

38. Wilhelm Núñez B. Propuesta de indicadores para la creación de un sistema de control de gestión para las unidades de procuramiento de órganos en Chile [Internet] [Memoria de Título]. Universidad de Chile; 2018. Disponible en: http://repositorio.uchile.cl/handle/2250/170051

39. Kefuri: Technology to increase detection of possible organ donors [Internet]. ResearchGate. [citado el 18 de julio de 2019]. Disponible en: https://www.researchgate. net/project/Kefuri-Technology-to-increase-detection-of-possible-organ-donors

40. Alcayaga Droguett R, Stiepovich Bertoni J, González Fuenzalida F, Alcayaga Droguett R, Stiepovich Bertoni J, González Fuenzalida F. Diseño de una propuesta de protocolo de estandarización y gestión de calidad para el proceso de procuramiento de órganos. Rev Med Chile 2019; 147 (3): 296-304. 